\title{
Unexpected kidney-restricted role for IL-17 receptor signaling in defense against systemic Candida albicans infection
}

\author{
Kritika Ramani,, Chetan V. Jawale, ${ }^{1}$ Akash H. Verma,, Bianca M. Coleman, ${ }^{1}$ Jay K. Kolls, ${ }^{2}$ \\ and Partha S. Biswas' \\ 'Division of Rheumatology and Clinical Immunology, University of Pittsburgh, Pittsburgh, Pennsylvania, USA. ${ }^{2}$ Richard \\ King Mellon Foundation for Pediatric Research, Children's Hospital of UPMC, Pittsburgh, Pennsylvania, USA.
}

\begin{abstract}
Kidney injury is a frequent outcome in patients with disseminated Candida albicans fungal infections. IL-17 receptor (IL-17R) signaling is critical for renal protection against disseminated candidiasis, but the identity and function of IL-17-responsive cells in mediating renal defense remains an active area of debate. Using BM chimeras, we found that IL-17R signaling is required only in nonhematopoietic cells for immunity to systemic C. albicans infection. Since renal tubular epithelial cells (RTEC) are highly responsive to IL-17 in vitro, we hypothesized that RTEC might be the dominant target of IL-17 activity in the infected kidney. We generated mice with a conditional

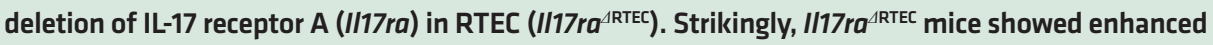
kidney damage and early mortality following systemic infection, very similar to $/ 117 \mathrm{ra}^{-/-}$animals. Increased susceptibility to candidiasis in II17ra ${ }^{\text {RTEC }}$ mice was associated with diminished activation of the renal protective Kallikrein-kinin system (KKS), resulting in reduced apoptosis of kidney-resident cells during hyphal invasion. Moreover, protection was restored by treatment with bradykinin, the major end-product of KKS activation, which was mediated dominantly via bradykinin receptor b1. These data show that IL-17R signaling in RTEC is necessary and likely sufficient for IL-17-mediated renal defense against fatal systemic $C$. albicans infection.
\end{abstract}

Conflict of interest: The authors have declared that no conflict of interest exists.

Submitted: October 20, 2017

Accepted: April 4, 2018

Published: May 3, 2018

Reference information: JCI Insight. 2018;3(9):e98241. https:// doi.org/10.1172/ji.insight.98241

\section{Introduction}

Candida albicans is a commensal fungus that causes severe disseminated infections $(1,2)$. Disseminated candidiasis is the third most common nosocomial infection in hospitalized patients, with a candidemia-attributable mortality rate ranging between $14.5 \%-49 \%(2-4)$. In most cases, autopsy studies revealed that kidneys are the major target organs following bloodstream $C$. albicans infection (5). Currently, there are no approved vaccines against any fungal pathogens. Moreover, antifungal drugs are administered too late in patients due to a lack of sensitive early diagnostic tools (6). Thus, the need for effective therapeutic strategies to counter this fatal infection is compelling and likely to increase with time. However, the development of new therapies is hindered by our lack of understanding of the host-pathogen interactions in driving protective antifungal immunity in the kidney.

Renal infections occur via hematogenous routes or from ascending spread from the bladder or urethra. The early innate response to invading $C$. albicans is mediated by a coordinated effort between the effectors of innate immunity and kidney-resident cells (5). This ensures successful clearance of fungal pathogens, followed by repair of damaged renal tissue. However, the cross-talk between the infiltrating immune cells and kidney-resident cells in shaping local anti-Candida immunity is a highly neglected area of inquiry.

In experimental models, IL-17A (IL-17) has emerged as a vital player of immunity against disseminated candidiasis (7-11). IL-17 signals through a receptor complex composed of IL-17RA and IL-17RC (12). However, our understanding of the downstream signaling events in IL-17 target cells in vivo is limited due to ubiquitous expression of the IL-17R and paucity of precise genetic tools to dissect IL-17 signaling in specific cell types. This has created major controversies in the IL-17 field, particularly in the area of anti-Candida immunity. For example, in oropharyngeal candidiasis (OPC), IL-17R signaling in oral epithelial cells drives the expression of antimicrobial peptides necessary for defense against candidiasis, and there is no role for the IL-17R signaling in the hematopoietic compartment (13). In disseminated candidiasis, we and others have shown that IL-17 is produced locally in the kidney upon infection and that kidney-resident cells 
respond to IL-17 directly $(7,14,15)$. In contrast, another study suggested that IL-17 is not made within the kidney but acts on NK cells to drive the production of GM-CSF, with protective activities in this setting of candidiasis (8). To date, the in vivo significance of IL-17R signaling in the kidney compartments where IL-17 mediates immunity against $C$. albicans is unknown.

IL-17 is classically recognized as a regulator of neutrophils and antimicrobial peptides. This is reflected in the profile of IL-17-induced genes in OPC, where genes encoding CXC chemokines, $\beta$-defensins, calprotectin (S100A8/9), and lipocalin 2 are upregulated (16). In a recent study from our laboratory, we identified the kallikreins $(K l k)$ as a distinct class of IL-17 target genes with renal protective functions in disseminated candidiasis (9). This unexpected observation highlighted that IL-17 induces expression of tissue-specific genes depending on target organs and disease context.

Tissue $K l k$, an essential component of $K l k$-kinin system (KKS), are a family of 15 related serine proteases. Klk1, in particular, plays a critical role in renal function and pathology (17). Klk act on kininogens to generate bradykinin and kallidin. Bradykinin signals through 2 G-protein coupled receptors: bradykinin receptor b2 (Bdkrb2) is constitutively expressed, and Bdkrb1 is induced in inflammatory conditions (17). Accordingly, mice lacking components of the KKS and humans with polymorphisms in KKS-related genes exhibit exacerbated kidney pathology in acute or chronic kidney diseases (18-22).

In disseminated candidiasis, progressive loss of kidney function is directly related to the inability of the host to clear the fungus and repair injured tissue. Renal tubular epithelial cells (RTEC) play a particularly pivotal role in driving inflammation in the kidney following acute or chronic injury $(23,24)$. RTEC constitute the majority among the cell types in the kidney cortex, where hyphal invasion and immune cells infiltration are hallmarks of early-stage infection, followed by spread to the medullary region at a later time point $(5,25)$. RTEC are activated by pathogen-associated molecular patterns and inflammatory mediators to secrete cytokines, chemokines, and tissue protective growth factors $(14,23,24)$. We have previously shown that Klk1 is induced in RTEC in an IL-17-dependent manner following systemic infection (9). However, the contribution of IL-17R signaling in RTEC in mediating renal immunity to $C$. albicans is poorly understood.

Here, we provide evidence indicating that the contribution of IL-17RA signaling in hematopoietic cells in systemic infection is not required during immunity against disseminated candidiasis. Rather, IL-17 produced locally in the kidney upon infection mediates nonredundant renal-protective signals through IL-17RA on RTEC. Consequently, mice deficient in IL-17RA signaling in RTEC (Il17ra ${ }^{A \mathrm{RTEC}}$ ) succumb to infection due to increased renal damage. Similar to $I l 17 \mathrm{ra}^{-/-}$mice, expression of kidney-protective Klk1 was markedly reduced in $1117 \mathrm{ra}^{\mathrm{ARTEC}}$ mice, and bradykinin-driven protection is mediated via Bdkrb1, not Bdkrb2. Our data indicate that IL-17R signaling in RTEC plays an essential role in activating renal-protective $\mathrm{KKS}$ in disseminated candidiasis.

\section{Results}

Innate TCR $\gamma \delta^{+} T$ cells are the primary source of IL-17 in the C. albicans-infected kidney. First, we confirmed whether renal expression of IL-17 family cytokines were upregulated in the early stages of disseminated candidiasis. When transcript expression of $I l 17 a, I l 17 f, I l 17 c$, and $I l 17 e$ were measured in the infected kidney at day 2 postinfection (p.i.), we observed a significant increase in $I l 17 a$ - but not $I l 17 f$, Ill7c, and Il17emRNA levels during disseminated infection (Figure 1A).

In the oral mucosa and skin, IL-17 from innate TCR $\alpha \beta^{+}$and $\mathrm{TCR} \gamma \delta^{+} \mathrm{T}$ cells are required for resistance to $C$. albicans infection $(26,27)$. To identify the cellular source of IL-17 in the kidneys, we took advantage of the sensitive $1117 \mathrm{a}^{\mathrm{Cre}}$ Rosa26R $\mathrm{R}^{\mathrm{eYFP}}$ (IL-17 ${ }^{\mathrm{eYFP}}$ ) fate-tracking mice that permanently marks IL-17-expressing cells with eYFP (28). These mice were systemically infected with $C$. albicans, and percentage of IL-17producing cells in the kidneys was determined based on eYFP expression at day 2 p.i. As expected, flow cytometry analysis revealed a 5 - to 6 -fold increase in the number of inflammatory cells $\left(\mathrm{CD} 45^{+}\right)$following infection (Figure 1B). When evaluated for IL-17 producers within the inflammatory cells, we observed a significant increase in the number of $\mathrm{eYFP}^{+}$cells in the $C$. albicans-infected kidneys than uninfected mice. We further gated on the $\mathrm{YFP}^{+}$cells to determine the absolute frequencies of IL-17-producing innate $\mathrm{TCR} \gamma \delta^{+}$and TCR $\alpha \beta^{+} \mathrm{T}$ cells in the kidneys. In uninfected mice, we saw a small baseline population of innate TCR $\gamma \delta^{+}$and TCR $\alpha \beta^{+}$T cells showing evidence of present or prior IL-17 production. Following infection, there is a 6- to 7-fold increase in the number of $\mathrm{CD} 45^{+} \mathrm{eYFP}{ }^{+} \mathrm{TCR} \delta^{+}$cells, indicating that innate $\mathrm{TCR} \gamma \delta^{+} \mathrm{T}$ cells expand and are the major source of IL-17 in the kidneys. While the frequency of IL-17producing innate TCR $\alpha \beta^{+}$cells was less compared with TCR $\gamma \delta^{+} \mathrm{T}$ cells, there was a reproducible 2-fold 
A

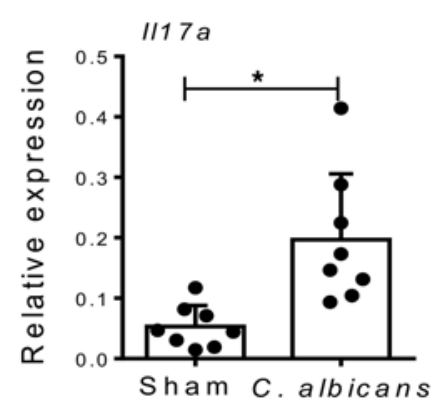

$1 / 17 f$

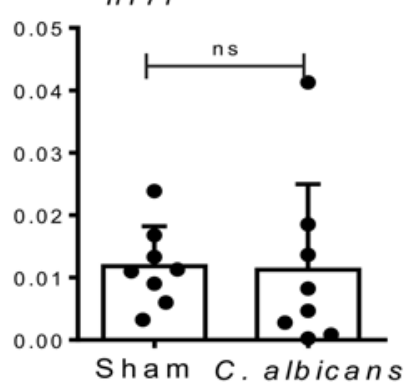

$117^{\circ} \mathrm{C}^{-}$

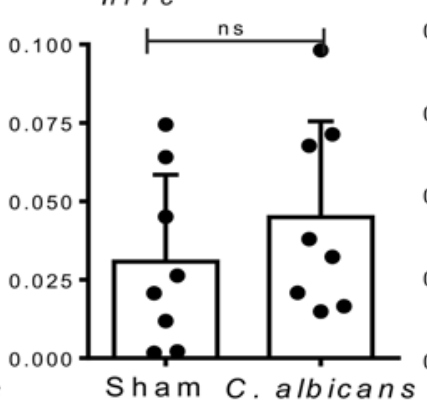

$1117 e$

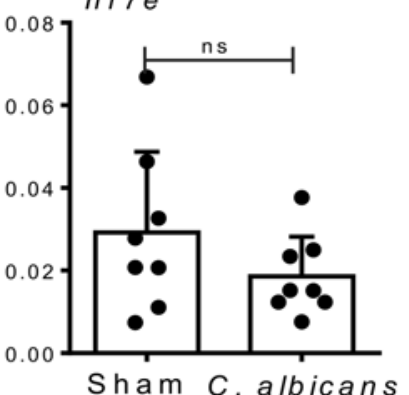

B
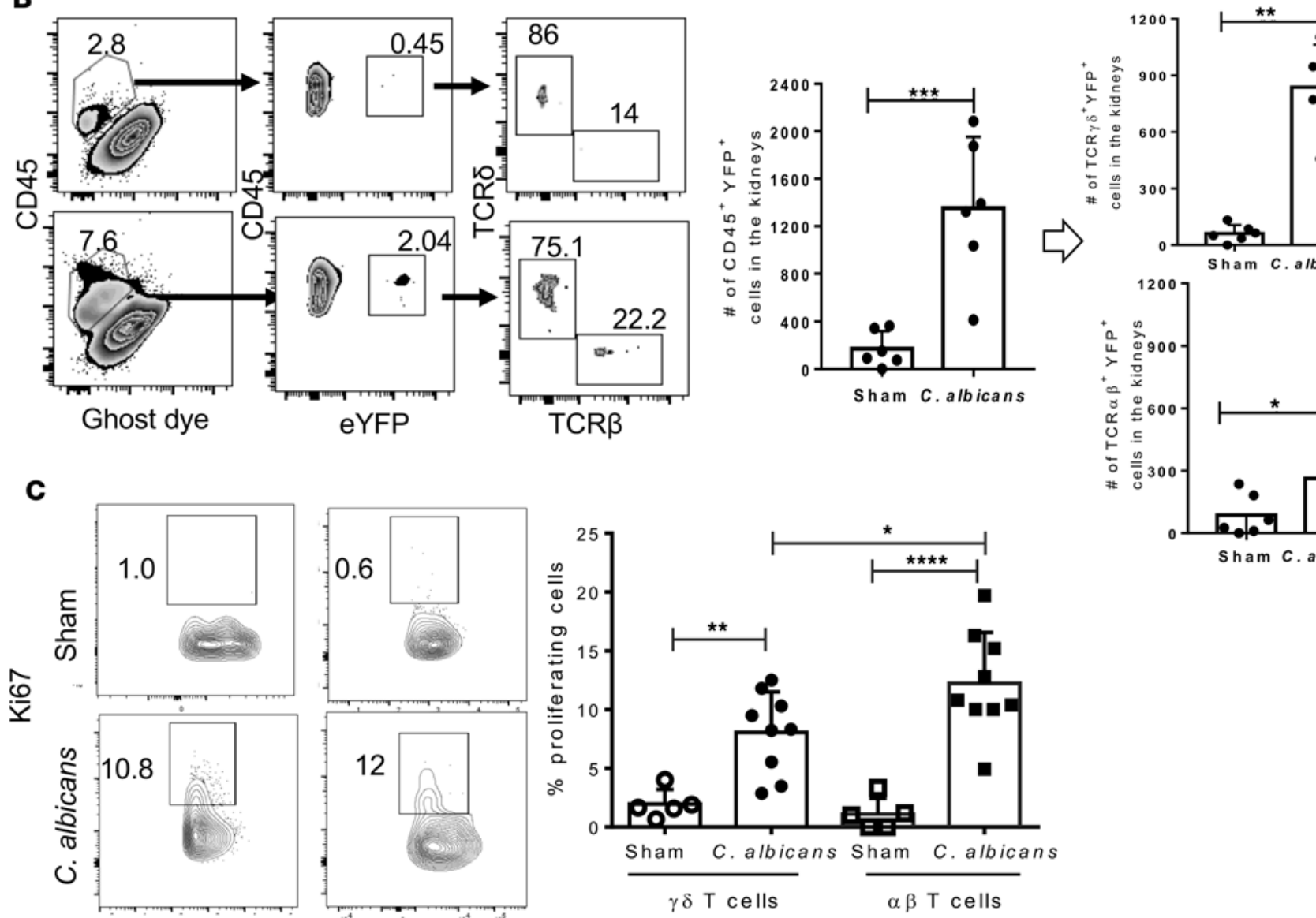

TCRס
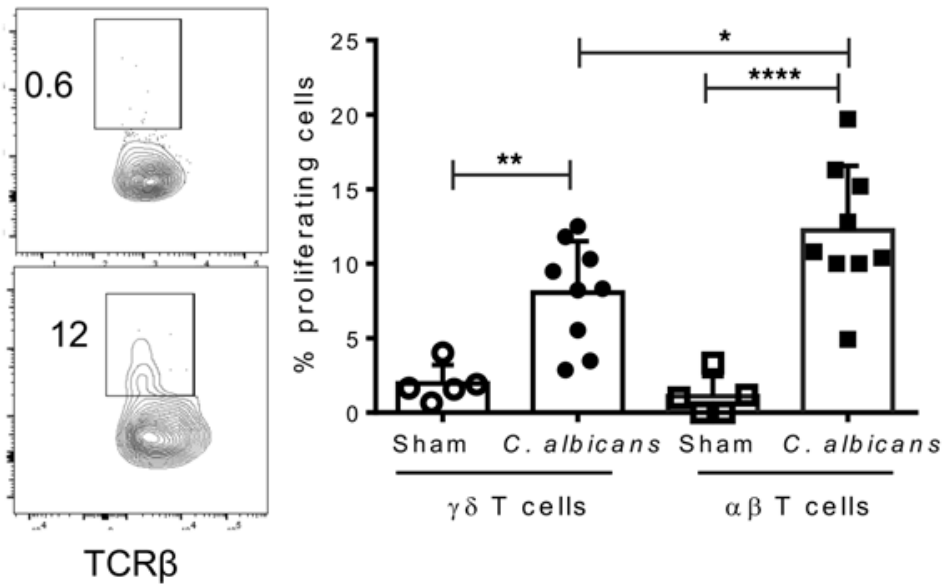

Figure 1. Innate TCR $\gamma \boldsymbol{\delta}^{+}$T cells produce IL-17 in C. albicans-infected kidney. (A) WT mice were either subjected to disseminated C. albicans infection or left uninfected (sham) $(n=8)$. At day 2 p.i., renal mRNA levels of II17a, II17f, II17c, and II17e were quantified by qPCR. (B) Single cell suspensions from the perfused kidneys of $C$. albicans infected or sham IL-17 ${ }^{\mathrm{eVF}}$ mice $(n=6)$ were used to determine the number of eYFP+ ${ }^{+}$cells (gated on live CD45 ${ }^{+}$) at day $2 \mathrm{p}$.i. Absolute numbers of innate TCR $\gamma \delta^{+}$and TCR $\alpha \beta^{+} \mathrm{T}$ cells producing IL-17 were evaluated by gating on live CD45+eYFP+ ${ }^{+}$cells. (C) Percentages of renal $\mathrm{Ki}-67^{+} \mathrm{TCR} \gamma \delta^{+}$and Ki-67+TCR $\alpha \beta^{+}$cells from C. albicans-infected $(n=9)$ or sham mice $(n=5)$ (gated on live CD45+ cells) at day 2 p.i. Numbers in the zebra (B) and contour (C) plots reflect percentages of cells. In the dot plots, each dot represents an individual mouse, and data are represented as mean \pm SD and compared by 2-tailed Student's $t$ test for $\mathbf{A}$ and $\mathbf{B}$ and by 1-way ANOVA for $\mathbf{C}$. Data are pooled from 2 independent experiments for $\mathbf{A}-\mathbf{C}{ }^{*} P<0.05$; ${ }^{* *} P<$ $0.01 ;{ }^{* *} P<0.001 ;{ }^{* * *} P<0.0001$.

increase in the number of TCR $\alpha \beta^{+}$cells in the infected kidneys compared with uninfected animals. This observation is in line with a previous report showing that IL-17-producing TCR $\gamma \delta^{+} \mathrm{T}$ cells play a vital role in the early host resistance against systemic $C$. albicans infection in the lung (29). Notably, we were unable to detect any eYFP-expressing ILC3 cells in the sham or infected kidney at this time point (data not shown).

To explain the increase in innate IL-17 producers in the fungal infected kidney, we next evaluated the proliferation of these cells by performing Ki-67 staining in non-fate-tracking mice. Systemic infection with $C$. albicans resulted in a strong proliferation of innate TCR $\gamma \delta^{+}$and TCR $\alpha \beta^{+} \mathrm{T}$ cells, as evidenced by the increased number of 
$\mathrm{Ki}-67^{+}$cells after infection (Figure 1C). The innate TCR $\alpha \beta^{+}$cells showed marginally increased proliferation than $\mathrm{TCR} \gamma \delta^{+} \mathrm{T}$ cells. These results indicate that kidney-resident or infiltrating innate $\mathrm{TCR} \gamma \delta^{+} \mathrm{T}$ cells are the primary cell types responsible for IL-17 production in the kidney during disseminated C. albicans infection.

IL-17RA signaling in nonhematopoietic cells is required for protection against disseminated candidiasis. Renal antifungal response is mediated by infiltrating cellular effectors (hematopoietic cells) and kidney-resident cells (nonhematopoietic cells). A previous study has shown that IL-17RA is required only in nonhematopoietic cells for oral defense against OPC (13). We hypothesized that there is likewise an essential contribution of IL-17RA in the nonhematopoietic compartment in disseminated candidiasis. To test this idea, BM cells from $I l 17 \mathrm{ra}^{-/-}\left(\mathrm{CD} 45.2^{+}\right)$ and WT $\left(\mathrm{CD} 45.1^{+}\right)$mice were adoptively transferred into lethally irradiated $1117 \mathrm{ra}^{-/-}$or WT recipients. Several studies have shown that most hematopoietic cells in the kidney are radiosensitive, thus allowing us to probe the contribution of hematopoietic vs. nonhematopoietic cells in renal defense against disseminated candidiasis (30-33). Baseline serum blood urea nitrogen (BUN), creatinine, and neutrophil gelatinase-associated lipocalin (NGAL) expression in the kidney revealed no overt difference in irradiation-induced kidney dysfunction or injury between WT and $I l 17 \mathrm{ra}^{-/-}$mice after BM reconstitution (Supplemental Figure 1, A and B; supplemental material available online with this article; https://doi.org/10.1172/jci.insight.98241DS1). Similar observations were also noted before the transfer of BM cells (24 hours after irradiation) between WT and $1117 \mathrm{ra}^{-/-}$mice (Supplemental Figure 1, C and D). Six weeks later, the recipient mice were tested for reconstitution by staining for CD $45.1^{+}$or CD $45.2^{+}$cells in the peripheral blood by FACS (Supplemental Figure 1E). Successfully reconstituted mice were subjected to disseminated candidiasis, and survival was evaluated over 14 days. $I l 17 \mathrm{ra}^{-/-}$mice reconstituted with $1117 \mathrm{ra}^{-/-} \mathrm{BM}$ succumbed to infection at an early time point (100\% mortality by day 7 p.i.), phenocopying $I l 17 \mathrm{ra}^{-/-}$mice (Figure 2A). WT mice receiving WT or $1117 \mathrm{ra}^{-/-} \mathrm{BM}$ exhibited increased survival (50\% survival at day 14 p.i.) than $I l 17 \mathrm{ra}^{-/-}$mice reconstituted with $\mathrm{Il1}_{7 \mathrm{ra}}{ }^{-1} \mathrm{BM}$, indicating that IL-17RA expression on hematopoietic cells is not required for antifungal defense. Interestingly, Il17 $\mathrm{ra}^{-1-}$ mice receiving WT BM showed increased susceptibility (100\% mortality around day 10 p.i.) in comparison with mice lacking Il17ra on hematopoietic cells. Remarkably, mortality in mice where IL-17RA signaling is absent in nonhematopoietic cells was almost similar to $I l 17 \mathrm{ra}^{-1-}$ mice reconstituted with $I l 17 \mathrm{ra}^{-/-} \mathrm{BM}$. These data establish that IL-17RA signaling is exclusively required in nonhematopoietic cells for antifungal immunity in disseminated candidiasis.

IL-17RA signaling in RTEC is critical for anti-Candida immunity in the kidney. The major kidney-resident cells implicated in renal inflammation belong to the glomerular, tubular, and vascular compartments, including podocytes, mesangial cells, endothelial cells, and proximal and distal RTEC (34). RTEC constitute approximately $70 \%$ of cells in the cortex, where they are in close proximity to C. albicans and inflammatory cells (34). RTEC express functional IL-17RA and produce cytokines and chemokines in response to IL-17 under in vitro condition $(14,24)$. Based on these findings, we sought to define the contribution of IL-17RA signaling specifically in RTEC during systemic infection. We generated mice lacking IL-17RA signaling in RTEC by crossing Il17ra ${ }^{f l / f l}$ mice to well-characterized $C d h 16^{C r e}\left(K s p 1.3^{C r e}\right)$ transgenic mice where the Cre recombinase is exclusively expressed in $\operatorname{RTEC}(35,36)$. Flow cytometry analysis of $I l 17 \mathrm{ra}{ }^{\text {flff }} \mathrm{C} d \mathrm{Ch} 16^{\mathrm{Cr}+}$ (henceforth referred to as $I l 17 \mathrm{ra}{ }^{4 \mathrm{RTEC}}$ ) mice confirmed effective deletion of IL-17RA in RTEC (CD45-CD133+) (Figure 2B). The kidney histology of Il17 $\mathrm{ra}^{\mathrm{ARTEC}}$ mice exhibit no overt changes in the renal architecture at the baseline (Supplemental Figure 2A). Overall, these data provided verification for the deletion of the Il17ra alleles in RTEC.

To assess the role of IL-17RA in RTEC, Il17ra ${ }^{4 R T E C}$ and $I l 17 \mathrm{ra}^{f l f f} \mathrm{Cdh} 16^{\mathrm{Cre}-}$ (hereafter referred to as control) mice were either infected with $C$. albicans or left uninfected, and survival was assessed over 14 days. $1117 \mathrm{ra}^{4 \mathrm{RTEC}}$ mice showed significantly increased susceptibility to systemic infection compared with control animals (Figure 2C). Nevertheless, $I l 17 \mathrm{ra}^{\triangle \mathrm{RTEC}}$ mice demonstrated marginally increased resistance to $1117 \mathrm{ra}^{-/-}$mice, indicating that IL-17RA signaling in kidney-resident cell types apart from RTEC may play a minor yet distinguishable role in renal immunity. Additionally, female and male mice demonstrated comparable susceptibility to disseminated candidiasis (Supplemental Figure 2B). Due to C. albicans strain-specific differences in antifungal immunity, we next assessed the susceptibility of $I l 17 \mathrm{ra}^{4 \mathrm{RTEC}}$ mice following systemic infection with a different strain of $C$. albicans (HUN96) (37). Il17ra ${ }^{4 R T E C}$ mice infected with HUN96 strain $\left(5 \times 10^{5} \mathrm{CFU}\right)$ showed early mortality compared with the control group (Figure 2D). To address the contribution of IL-17 signaling in other renal epithelial cell types, we generated mice lacking $I l 17 \mathrm{ra}$ in podocytes by crossing $I l 17 \mathrm{ra}{ }^{f l / l}$ mice to $N p h 2^{C r e}$ transgenic mice, in which Cre recombinase is solely expressed in podocytes (termed $I l 17 r^{4 \mathrm{POD}}$ ) (38). Unlike $I l 17 \mathrm{ra}^{\lrcorner \mathrm{RTEC}}$ mice, there was no difference in susceptibility between $I l 17 \mathrm{ra}{ }^{\lrcorner \mathrm{POD}}$ and control animals to disseminated candidiasis (Figure 2E). These results indicate that IL-17RA signaling in RTEC play an important and likely sufficient role for renal protection against disseminated candidiasis. 
A

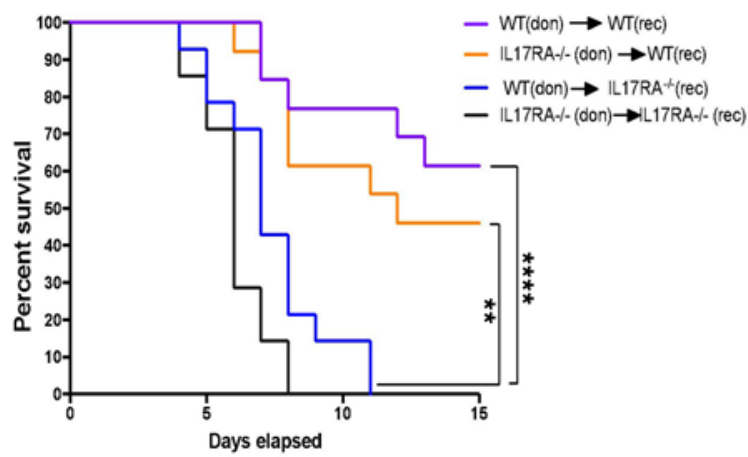

D

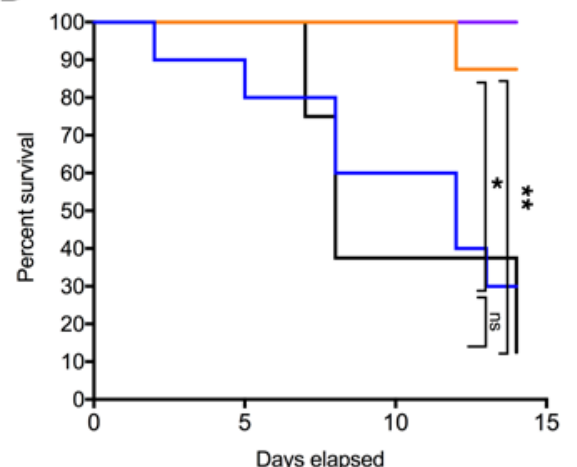

B

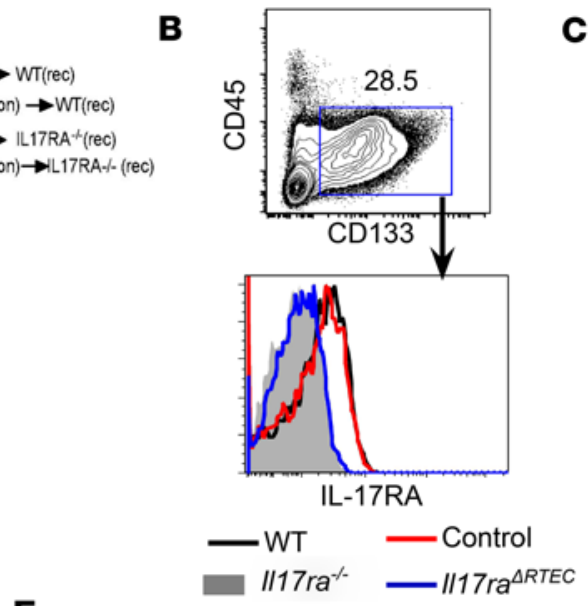

\section{E}

C

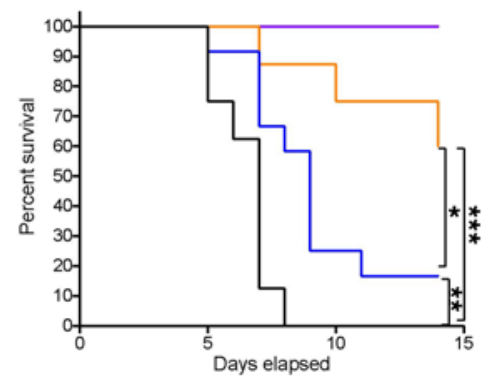

- II17rat
- Control
- II17raRTEC
- Sham

$$
\begin{array}{l|l}
\text { - Control } & \text { C. albicans } \\
\text { - II17raARTEC } & \text { HUN96 }
\end{array}
$$$$
\text { - Sham }
$$

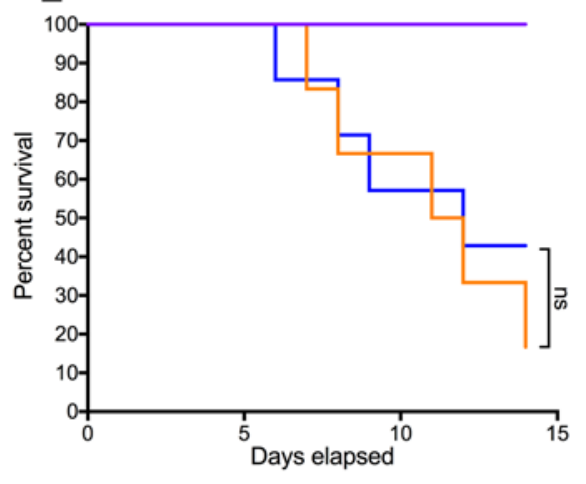

$\left.\begin{array}{l}\text { - II17ra }{ }^{\triangle P O D} \\ \text { - Control } \\ \text { - Sham }\end{array}\right]$ C. albicans

Figure 2. RTEC-specific expression of IL-17RA is required for antifungal immunity in the kidney. (A) BM cells from II17ra $\mathrm{ra}^{-1-}$ (CD45.2+) and WT (CD45.1 $)$ mice were adoptively transferred into irradiated ( $9 \mathrm{~Gy}) I 117 \mathrm{ra}^{-1-}$ or WT recipients $(n=14)$. Six weeks later, mice were systemically infected with $C$. albicans, and survival was evaluated over 14 days. Don, donor; rec, recipients. (B) Total kidney cells from WT, $\| 117 \mathrm{ra}^{-1}$, control, and $I / 17 \mathrm{ra}{ }^{4 \mathrm{RTEC}}$ mice $(n=3)$ were evaluated for IL-17RA expression on RTEC (live CD45-CD133+). The number in the contour plot reflects percentage of cells. Histogram is representative of 1 of 3 independent experiments. (C) Control, II17ra ${ }^{\text {RTEC}}$, and II17 ra-/- mice were systemically infected with C. albicans (CAF2-1 strain; $1 \times 10^{5}$ CFU) ( $\left.n=15-16\right)$ or left uninfected $(n=3-5)$. Mice were evaluated for survival over 14 days. (D) Control, I/17ra ${ }^{A R T E C}$, and I/17ra ${ }^{-/-}$mice were systemically infected with C. albicans

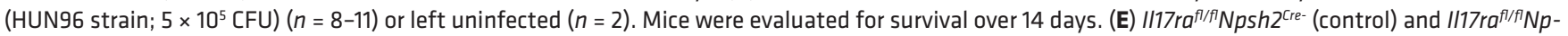
sh $2^{\text {Cre+ }}\left(I / 17 \mathrm{ra}^{4 \mathrm{POD}}\right)$ mice were either subjected to infection $(n=8-10)$ or left uninfected $(n=3)$ and were evaluated for survival over 14 days. Data are pooled from 3 independent experiments for $\mathbf{A}, \mathbf{C}$, and $\mathbf{E}$ and 2 independent experiments for $\mathbf{D}$. The comparison of survival curves were performed by Log-rank (Mantel-Cox) test. ${ }^{*} P<0.05 ;{ }^{* *} P<0.01 ;{ }^{* *} P<0.001 ;{ }^{* * *} P<0.0001$.

$N K$ cells are functionally normal in mice with RTEC-specific deletion of IL-17RA. A previous report has suggested a critical role for IL-17RA in NK cells mediated antifungal immunity following disseminated candidiasis (8). In this study, $I l 17 \mathrm{ra}^{-/-}$mice demonstrated increased fungal load in the kidney due to a cell-intrinsic defect in the development of functional NK cells. Although $C d h 16^{C r e}$ transgenic mice has been widely used for RTEC-specific gene targeting (39), we wanted to rule out any defects in NK cell function due to leaky expression of Cre recombinase that could account for the increased susceptibility of $I 117 \mathrm{ra}{ }^{4 \mathrm{POD}}$ mice to fungal infection. Flow cytometry analysis of BM cells showed comparable IL-17RA expression on immature NK (iNK) and precursor NK (NKP) cells between $I l 17 r a^{A \mathrm{POD}}$ and control mice (Figure 3A and Supplemental Figure 3).

We further assessed the functionality of NK cells from $I l 17 \mathrm{ra}^{\triangle \mathrm{RTEC}}$ and control mice by measuring IFN $\gamma$ production from splenic NK cells following LPS injection. In line with the previous report (8), NK cells

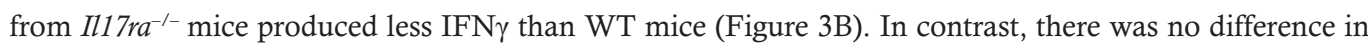
the percentage of IFN $\gamma$-producing NK cells between the $I l 17 \mathrm{ra}{ }^{\triangle \mathrm{RTEC}}$ and control animals. Notably, we were unable to detect IFN $\gamma$ or GM-CSF from splenic NK cells in mice following systemic $C$. albicans infection 
at 24 hours p.i. in our experiments (Supplemental Figure 4, A and B). These results suggest that increased susceptibility of $I l 17 \mathrm{ra}^{4 \mathrm{RTEC}}$ mice to disseminated candidiasis is not due to defects in NK cell function.

Kidney fungal clearance is moderately impaired in mice with RTEC-specific deletion of IL-17RA. We next determined the impact of RTEC-specific IL-17RA signaling in fungal clearance and early inflammatory events in the kidney. $I l 17 \mathrm{ra}^{\lrcorner \mathrm{RTEC}}$ and control kidney surprisingly exhibited comparable fungal load at day 2 p.i., a time point at which $I l 17 \mathrm{ra}^{-/-}$mice showed increased fungal burden (Figure 4A) (9). However, Ill $7 \mathrm{ra}^{4 \mathrm{RTEC}}$ mice showed half a log increase in the fungal load compared with control kidney at day 5 p.i. These results indicate that IL-17RA signaling in RTEC marginally affects fungal clearance, albeit at a delayed time point.

We also compared the frequency of kidney-infiltrating innate cells in the $1117 \mathrm{ra}{ }^{\mathrm{ARTEC}}$ and control mice following fungal infection. In agreement with the fungal clearance data, the percentages of kidney-infiltrating total inflammatory cells $\left(\mathrm{CD} 45^{+}\right)$, neutrophils, macrophages, and NK cells were comparable between the groups at day 2 p.i. (Figure 4B). Renal transcript expression of Cxcl5, S100a8, and Defb1 was significantly downregulated in the kidney of $I l 17 \mathrm{ra}^{4 \mathrm{RTEC}}$ mice (Figure 4C). However, other IL-17-responsive genes such as Lcn2 and CXcl1 showed no change in the Il17ra ${ }^{4 \mathrm{RTEC}}$ mice, similar to what has been shown in $I l 17 \mathrm{ra}^{-1-}$ mice (Supplemental Figure 5) (9). These data suggest that reduced expression of antimicrobial peptides in $I 117 \mathrm{ra}^{4 \mathrm{RTEC}}$ mice may account for the defect in fungal clearance at a delayed time point.

IL-17RA signaling in RTEC prevents kidney damage following disseminated candidiasis. Based on our survival data (Figure 2C), we wanted to determine whether the absence of IL-17RA signaling in RTEC is associated with increased renal damage, leading to loss of kidney function. H\&E staining of serial kidney sections revealed increased tissue pathology in the cortex and inner and outer medullary regions of $I l 17 \mathrm{ra}^{4 \mathrm{RTEC}}$ at day 7 p.i. (Figure 5A and Supplemental Figure 5B). Consequently, renal function was compromised in $1117 \mathrm{ra}^{\lrcorner \mathrm{RTEC}}$ mice as evident from increased serum BUN levels at day 7 p.i. (Figure 5B). Il17ra ${ }^{4 \mathrm{RTEC}}$ kidney demonstrated a marked increase in the expression of NGAL and kidney-injury marker 1 (KIM1), classical kidney injury markers (Figure 5C, Supplemental Figure 5B, and Supplemental Figure 6, A and B). Collectively, these results demonstrate that IL-17RA signaling in RTEC prevents kidney damage during disseminated candidiasis.

We have previously shown that IL-17RA signaling alleviates renal injury during hyphal invasion by preventing the apoptosis of kidney-resident cells (9). Therefore, we compared the apoptosis of tubular epithelial cells between $I l 17 \mathrm{ra}^{4 \mathrm{RTEC}}$ and control kidneys following $C$. albicans infection. At day 7 p.i., we observed a significantly increased frequency of apoptotic kidney-resident tubular epithelial cells (CD45 $\mathrm{CD}_{133^{+}}$) in $I l 17 \mathrm{ra}^{4 \mathrm{RTEC}}$ mice compared with control kidney (Figure 6A). Supporting our apoptosis data, absence of IL-17RA in RTEC also resulted in a striking increase in the number of active Caspase- $3^{+}$tubular epithelial cells $\left(\mathrm{CD} 45^{-} \mathrm{CD} 133^{+}\right.$) (Figure 6B). Thus, activation of IL-17RA signaling in RTEC limits renal damage during systemic fungal infection by limiting the apoptosis of kidney-resident cells.

IL-17RA-dependent expression of Klk1 in RTEC protects mice against systemic $C$. albicans infection. Our published study identified an intriguing link between IL-17/KKS axis-mediated renal protection against disseminated candidiasis (9). We were the first to our knowledge to show that kidney-protective Klk1 is expressed only in RTEC following fungal infection in an IL-17-dependent manner (9). To confirm that Klk1 is indeed produced specifically from RTEC, we measured the transcript expression of Klk1 in the kidneys of infected $I l 17 \mathrm{ra}^{4 \mathrm{RTEC}}$ and control mice. Similar to what has been shown in $1117 \mathrm{ra}^{-1-}$ mice (9), $I 117 \mathrm{ra}^{\triangle \mathrm{RTEC}}$ kidney demonstrated a significant reduction in the mRNA levels of Klk1 and Klk1b26 compared with control mice at day 2 p.i. (Figure 7A). Consequently, RTEC in the cortex and medullary region of the infected kidney expressed diminished levels of Klk1 in the absence of IL-17RA at day 7 p.i. (Figure 7B and Supplemental Figure 6C). These results highlight the essential contribution of IL-17RA signaling in RTEC for the production of renal-protective $\mathrm{K} 1 \mathrm{k} 1$ in disseminated infection.

Klk1 mediates cleavage of kininogens to generate bradykinin, which signals through Bdkrb1 and Bdkrb2 (17). To define the role of RTEC-derived K1k1 in renal immunity, Il17ra ${ }^{\mathrm{RTEC}}$ mice were either treated with bradykinin or left untreated, and survival was evaluated following systemic infection. Bradykinin-treated $I l 17 \mathrm{ra}^{\mathrm{ARTEC}}$ mice exhibited significantly increased survival benefit in comparison with the untreated group (Figure 7C). Collectively, these results extend our previous finding and indicate that the IL-17/Klk1 axis in RTEC plays a critical role in the renal host defense in disseminated candidiasis.

We next wanted to define the individual contribution of Bdkrb1 and Bdkrb2 in renal defense against systemic fungal infection. First, we evaluated $B d k r b 1$ and $B d k r b 2 \mathrm{mRNA}$ expression in the infected kidney of WT mice. The fungal infection resulted in a significant upregulation of $B d k r b 1$ but not $B d k r b 2$ transcript expression at day 2 p.i. (Figure 7D). This result indicates that the inflammatory milieu in the kidney contributes to the induction 
A
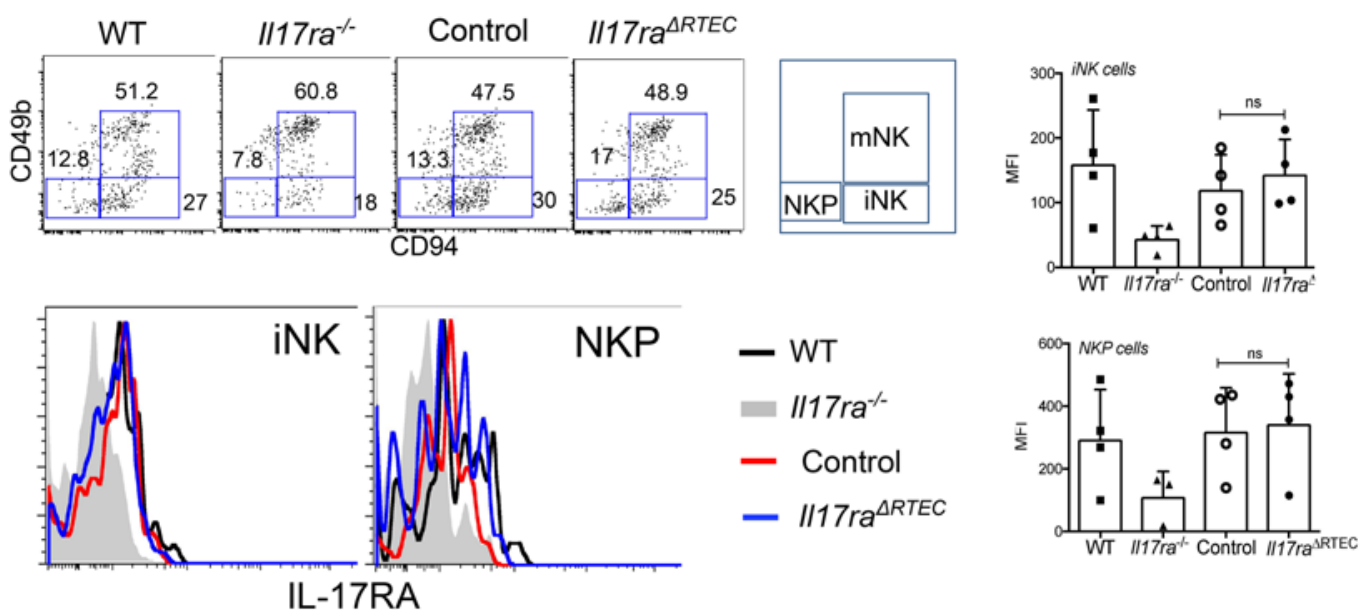

B

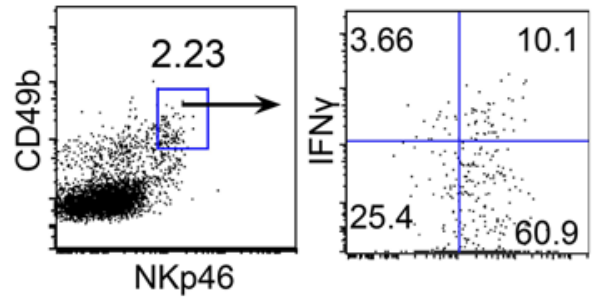

Control

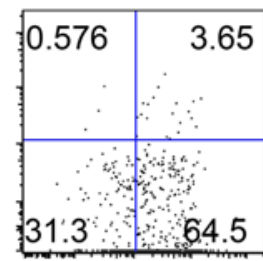

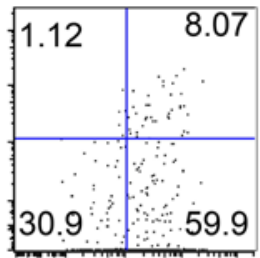

59.9

CD69

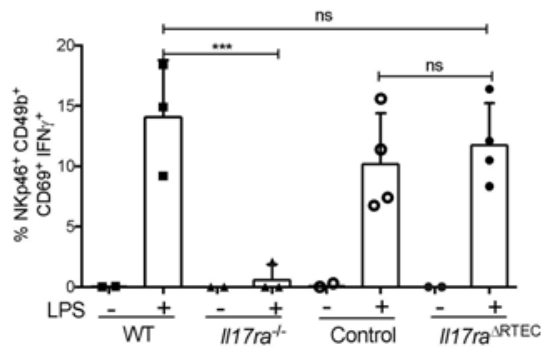

Figure 3. NK cells are functional in II17raA $R$ TEC mice. (A) BM cells from WT, II17ra ${ }^{-/}$, control, and II17ra ${ }^{A R T E C}$ mice $(n=4)$ were evaluated for IL-17RA expression on mature NK (mNK) (liveCD45+CD3-NKC2d+CD49b+CD94+), immature NK (iNK) (liveCD45+CD3-NKC2 $\mathrm{d}^{+}$CD49b-CD94+), and precursor NK (NKP) (liveCD45+CD3-NKC2 $\left.\mathrm{d}^{+} \mathrm{CD} 49 \mathrm{~b}^{-} \mathrm{CD} 94^{-}\right)$cells by flow cytometry. (B) WT, I/177 $\mathrm{ra}^{-/}$, control, and I/17ra ${ }^{4 \mathrm{RTEC}}$ mice were either injected i.p. with LPS (1.5 ng/ml) $(n=4)$ or left untreated $(n=2)$. Five hours later, splenic NK cells (gated on liveCD45+CD3-NKp46+CD49b+CD69+) were evaluated for IFN $\gamma$ production by intracellular cytokine staining. Numbers in the dot plot indicate percentages of cells. In the dot plots, each dot represents individual an mouse, and data are represented as mean \pm SD. Data were pooled from 3 and 2 independent experiments for $\mathbf{A}$ and $\mathbf{B}$, respectively, and analyzed by 1 -way ANOVA. ${ }^{* * *} P<0.001$.

of Bdkrb1 expression, similar to what has been shown in other inflammatory kidney diseases (18). To determine the specific role of Bdkrb1 and Bdkrb2 in IL-17/Klk1 axis-driven renal defense, anti-Bdkrb1- or anti-Bdkrb2 antagonist-treated WT mice were subjected to disseminated candidiasis, and survival was evaluated over 14 days. Mice treated with selective Bdkrb1 antagonist succumb to infection earlier (100\% mortality around day 7 p.i.) compared with the untreated or Bdkrb2 antagonist-treated groups (Figure 7E). The survival of Bdkrb2 antagonist-treated mice was comparable with untreated mice. Collectively, these data suggest that Bdkrb1 activation is a key downstream mediator in the IL-17/Klk1-mediated protection against disseminated candidiasis.

\section{Discussion}

While it is evident that IL-17 is needed for renal immunity to candidiasis, the underlying mechanisms are poorly understood. In the past decade, most studies in the IL-17 field have focused on the generation of IL-17-producing cells. With the development of mice with conditional deletion of IL-17R, studies defining the downstream effects of IL-17 on target cells have just begun to appear. For example, oral epithelial cells drive IL-17R-dependent responses against OPC through regulation of antimicrobial peptides (13). On the other hand, IL-17R sig- 
A

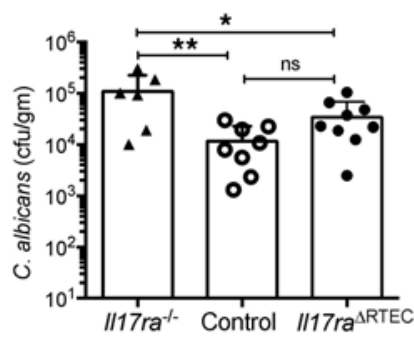

Day 5 p.i.

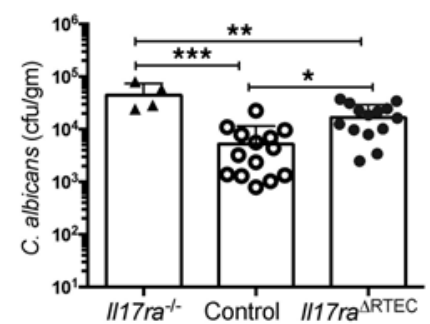

C

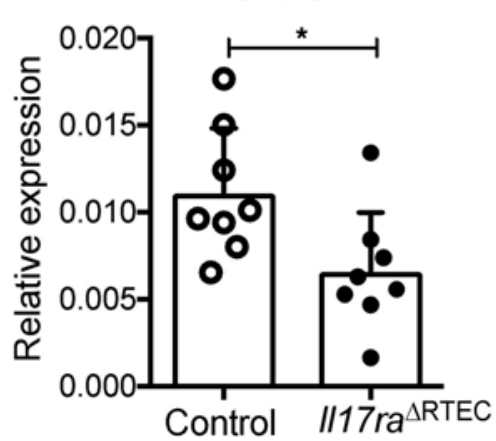

B

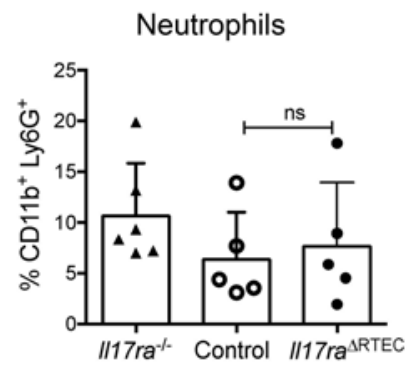

Macrophages
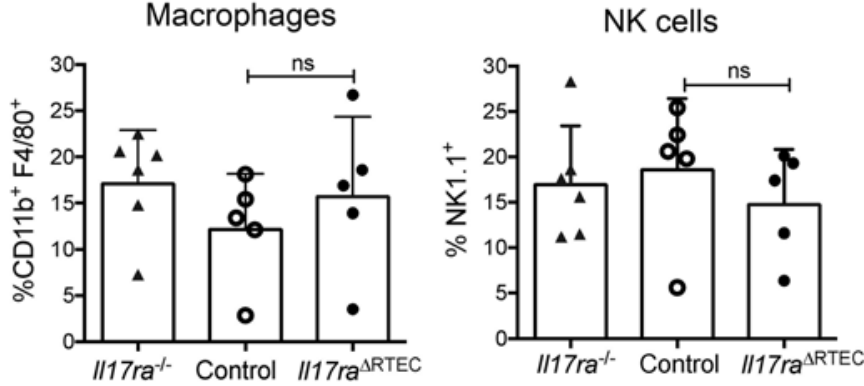
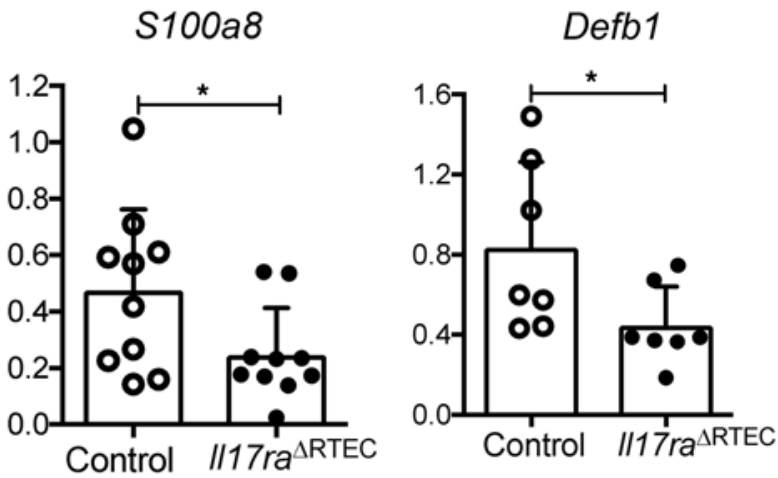

Figure 4. Early renal innate response in $I / 17 \mathrm{ra}^{4 \mathrm{RTEC}}$ mice after disseminated candidiasis. Control, II17ra ${ }^{4 \mathrm{RTEC}}(n=8-14)$ and $/ 117 \mathrm{ra}{ }^{-/-}$mice $(n=6)$ were subjected to systemic $C$. albicans infection. (A) Fungal burden was measured in the kidneys at days 2 and 5 p.i. Data for surviving $/ 177 \mathrm{ra}^{-/-}$mice $(n=4)$ are shown for day

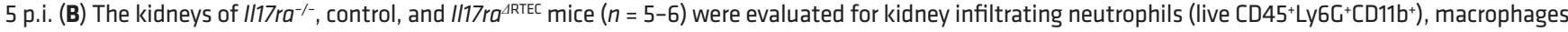
(live CD45+F4/80+CD11b+), and NK cells (live CD45+CD3-NK1.1+) at day 2 p.i. (C) Renal transcript expression of Cxcl5, S100a8, and Defb1 was measured by qPCR in control and I/17ra ${ }^{A R T E C}$ mice $(n=7-10)$ at day 2 p.i. Each dot represents an individual mouse, and data are represented as mean \pm SD. Data are pooled from 2 independent experiments for $\mathbf{A}$ and $\mathbf{B}$ and 3 independent experiments for $\mathbf{C}$ and were analyzed by 1-way ANOVA. ${ }^{*} P<0.05 ;{ }^{* *} P<0.01$; ${ }^{* * *} P<0.001$.

naling in the lung epithelium establishes chemokine gradients essential for antibacterial immunity (40). The common emergent theme from these studies is that IL-17-responsive genes are differentially regulated in similar cell types from different organs, with profound implications in designing organ-targeted therapeutic strategies to combat systemic fungal infections. Thus, lessons from mucosal sites do not necessarily translate to solid organs, such as the kidney. Here, we show that IL-17R signaling in RTEC drives the activation of KKS, critical for renal protection against fungal invasion. Despite being a fatal systemic infection, the IL-17R signaling is markedly restricted in the kidney and not in the cells of immune system during antifungal immune response. This observation contrasts with $\mathrm{TNF} \alpha$, where activities on neutrophils and other myeloid cells are required for antifungal immunity (41). The study presented here is the first systematic dissection of cytokine signaling in the kidney to our knowledge and reveals insights into the renal activities of IL-17 in antifungal immunity.

IL-17RA serves as a receptor for several IL-17 family members, including IL-17, IL-17F, IL-17C, and IL-17E (12). Therefore, the increased susceptibility observed in Il17ra ${ }^{A T E C}$ mice could be due to defective signaling of several IL-17 family members other than IL-17. Since IL-17F, IL-17C, and IL-17E were unable to induce Klk1 expression in RTEC (9), it is unlikely that these members contribute to the protection in 


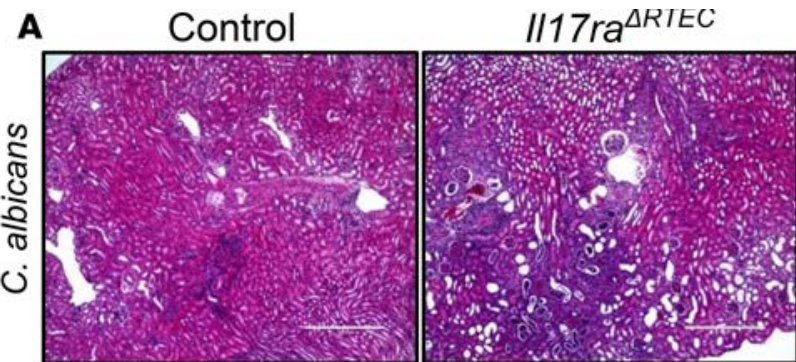

C
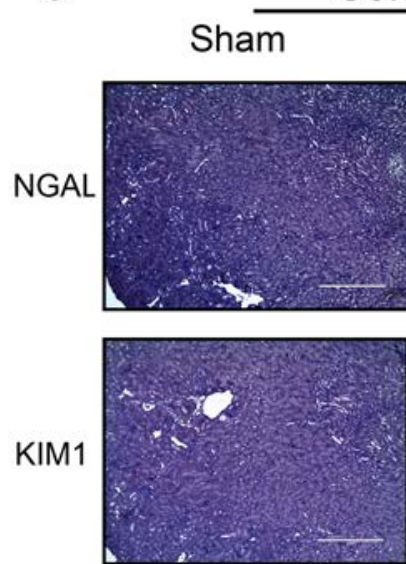

Control C. albicans
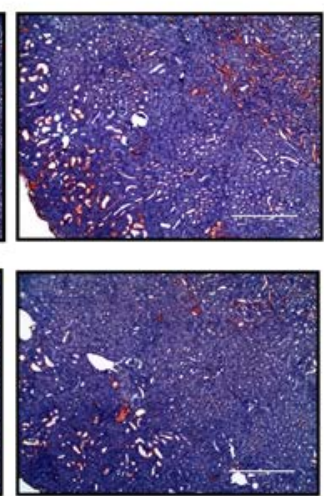

B

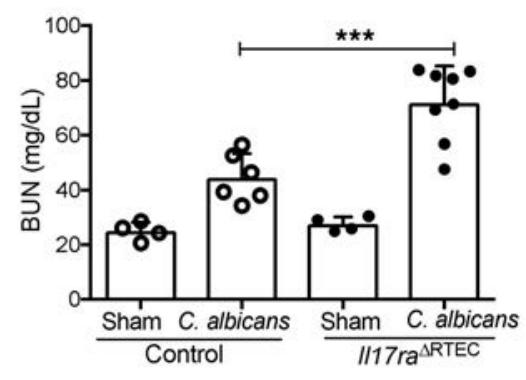

II17ra $\triangle R T E C$
C. albicans
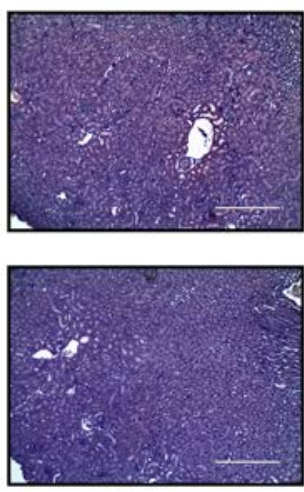
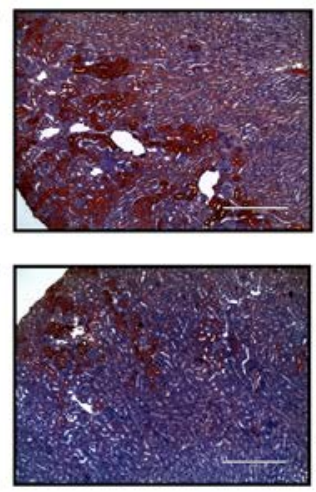

Figure 5. II17ra ${ }^{\text {ARTEC }}$ mice demonstrated aggravated renal damage following systemic $C$. albicans infection. Control and II17ra ${ }^{\text {ARTEC }}$ mice were either subjected to systemic C. albicans infection $(n=6-8)$ or left uninfected $(n=4)$. At day 7 p.i., mice were evaluated for (A) histopathology and inflammatory cell influx by H\&E staining (original magnification, 100x); (B) serum BUN level at day 7 p.i.; and (C) NGAL and KIM1 expression by IHC. Representative photomicrographs from 2 independent experiments for $\mathbf{A}$ and $\mathbf{C}$. Original magnification, 100x. Pooled results from 2 independent experiments for $\mathbf{B}$. Each dot represents an individual mouse, and data are represented as mean \pm SD and analyzed by 1-way ANOVA. ${ }^{* *} P<0.001$. Scale bars: $400 \mu \mathrm{m}$.

fungal infection. However, there is lack of conclusive data using targeted genetic approaches to rule out the potential involvement of IL-17C signaling in RTEC. Future studies with mice harboring RTEC-specific deletion of IL-17RE, an IL-17 receptor subunit that is solely utilized by IL-17C, will shed light on the role of this cytokine in antifungal immunity in the kidney (42).

A published study suggests that IL-17 may be important for driving NK cell function in disseminated infection through a mechanism dependent on GM-CSF-mediated neutrophil killing of C. albicans (8). Subsequently, a recent report has highlighted the essential role of type I IFN-driven IL-15 from inflammatory monocytes in inducing GM-CSF production from NK cells, necessary to enhance anti-Candida activity of neutrophils (43). However, discrepancies exist regarding the role of NK cells in disseminated infection. Quintin et al. showed that NK cells are redundant for antifungal defense in immunocompetent hosts (44). In fact, NK-depleted animals showed increased survival compared with the control group. Additionally, NK-depleted mice with severe combined immunodeficiency did not show enhanced susceptibility to systemic $C$. albicans infection (45). Although NK cells lack direct candidacidal capacity $(46,47)$, these cells can activate phagocytes by secreting IFN $\gamma(48,49)$. Interestingly, $C$. albicans suppresses NK cell function via induction of myeloid-derived suppressor cells, thus questioning the functional stability of NK cells in this system $(50,51)$. It is still possible that NK cells contribute to neutrophil-mediated early fungal clearance in the kidney, as reported by Bär et al. (8). However, experimental data showing a head-to-head comparison between cells of hematopoietic and nonhematopoietic origin deficient in IL-17R in anti-Candida immunity are currently lacking. Using BM chimera mice, we now show that the contribution of IL-17R signaling in hematopoietic cells is quite minimal in antifungal immunity. Accordingly, mice deficient in IL-17RA signaling in RTEC exhibited normal NK cell function, indicating that increased susceptibility of $1117 \mathrm{raATEC}$ 

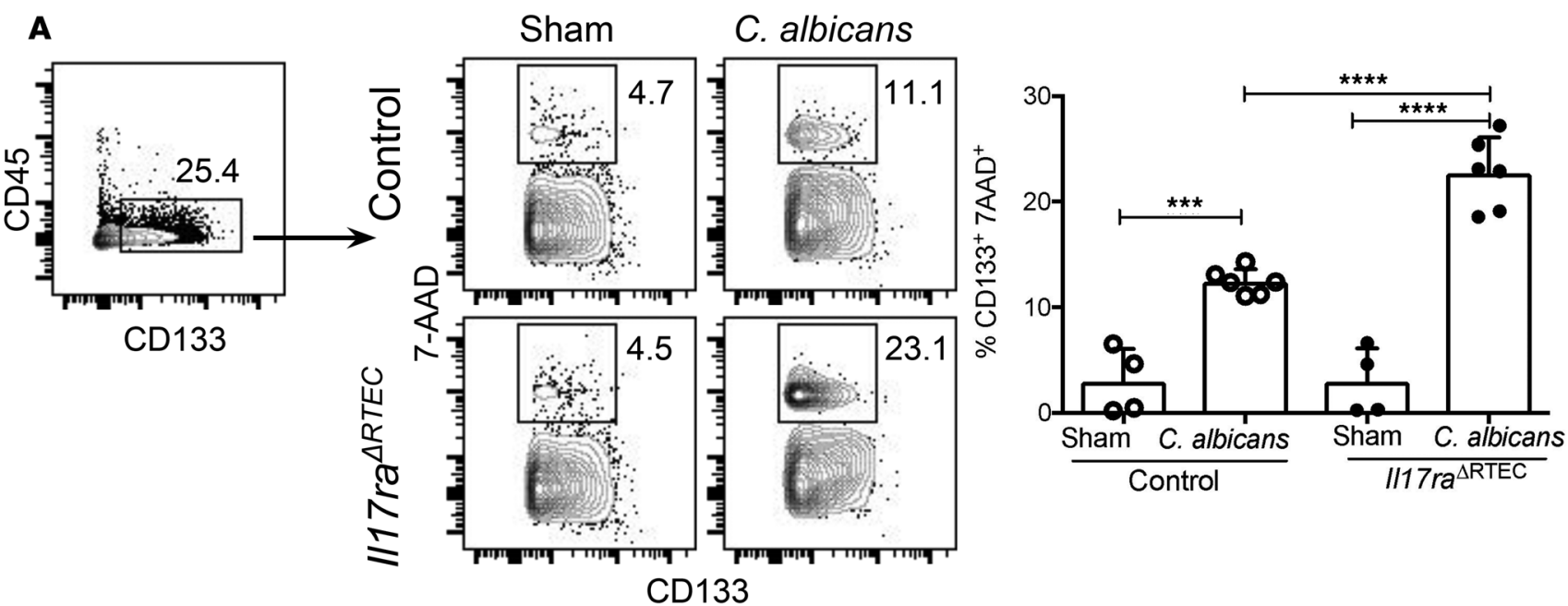

B
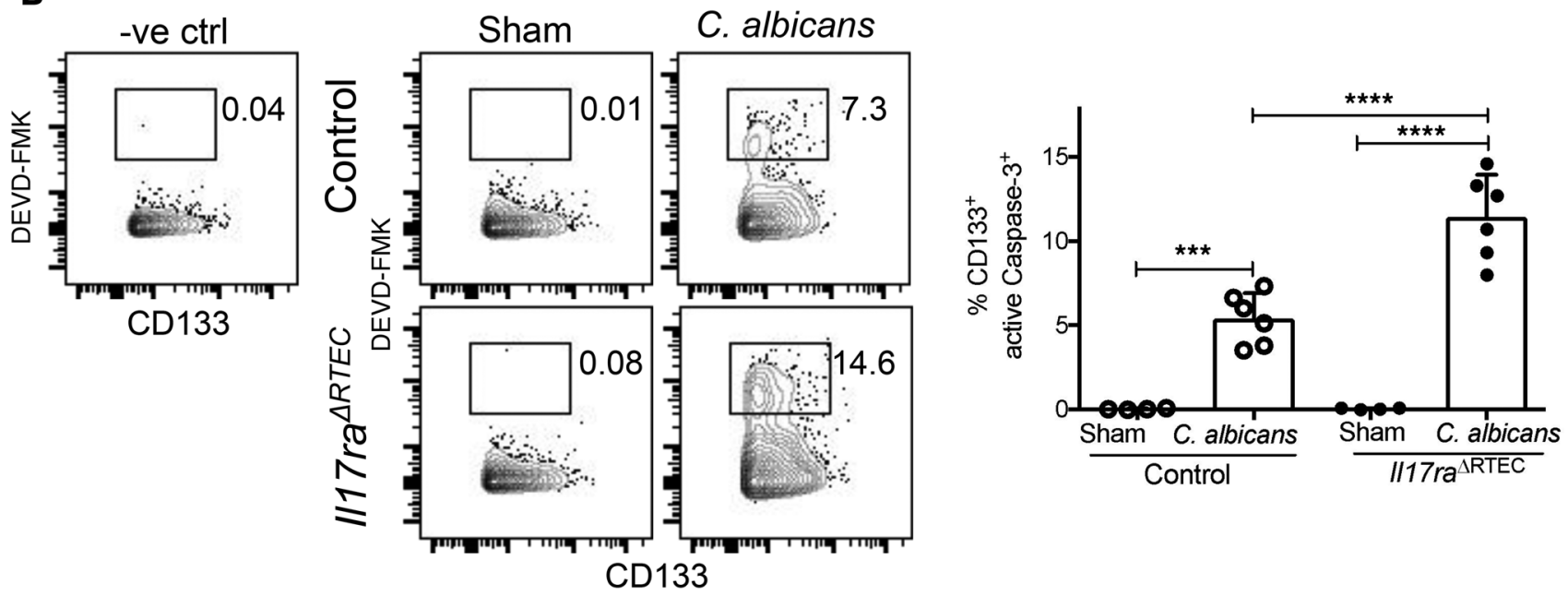

Figure 6. Increased apoptosis of RTEC in the absence of IL-17RA signaling following disseminated candidiasis. Control and I/17ra ${ }^{4 \mathrm{RTEC}}$ mice were subjected to systemic $C$. albicans infection $(n=6)$ or left uninfected $(n=4)$. The percentages of $(\mathbf{A})$ apoptotic kidney-resident tubular epithelial cells (gated on CD45-CD133+cells) were detected by 7-AAD staining, and (B) percentages of active Caspase- $3^{+}$kidney-resident tubular epithelial cells (gated on CD45 ${ }^{-}$ $\mathrm{CD}_{133^{+}}$cells) were quantified by DEVD-FMK staining. Numbers in the contour plot reflect percentages of cells. In the dot plots, each dot represents an individual mouse, and data are represented as mean \pm SD. Pooled data are from 2 independent experiments and analyzed by 1 -way ANOVA. ${ }^{* * *} P<0.001$; ${ }^{* * * *} P<0.0001$.

mice to disseminated infection is not due to NK cell defect. Although $1117 \mathrm{ra}^{-1-}$ mice demonstrated a defect in NK cell function, we were unable to detect any IFN $\gamma$ or GM-CSF production from NK cells following systemic $C$. albicans infection. The apparent discordant between our findings and others are poorly understood. It is possible that differences in the microbiome between animal colonies may account for the dissimilarities observed and requires careful consideration in the future.

RTEC constitute the major cell types (70\%) in the renal cortex, the site for hyphal invasion and immune response in the course of a systemic infection (34). RTEC express various pattern recognition and cytokine receptors and are, thus, uniquely positioned to interact with both pathogen and immune cells (34). We previously showed that RTEC are responsive to IL-17 (14). IL-17 in synergy with TNF $\alpha$ induced the expression of Klk1 in RTEC, but not in glomerular and vascular cells (9). However, there is a paucity of data using targeted genetic approaches that can identify specific cell types responsible for Klk1 production. We now show that mice with conditional deletion of IL-17RA in RTEC express diminished Klk1 and succumb to infection earlier than control animals. Interestingly, $I 117 \mathrm{ra}^{-1-}$ mice demonstrated a more severe phenotype than mice with conditional deletion of IL-17RA in RTEC. Since expression of the Cdh16 promoter varies between RTEC of proximal and distal tubules, it is possible that Cdh16 $6^{\text {Cre }}$ recombines and deletes IL-17RA in the majority but not all RTEC (35). Additionally, the absence of IL-17R signaling in podocytes, an epithelial cell type in 
A
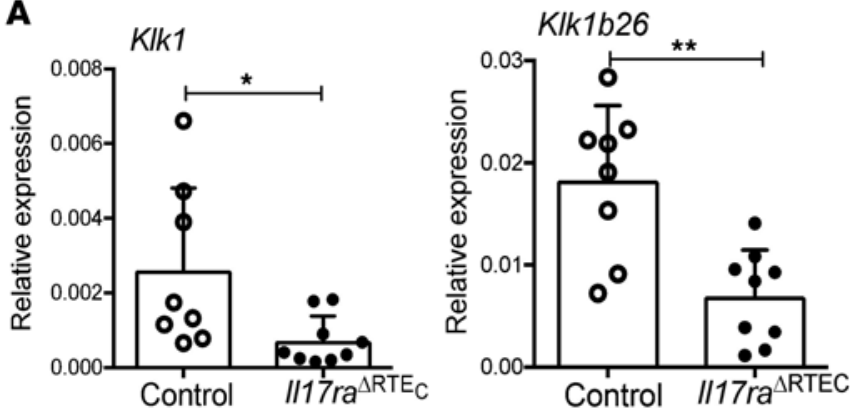

C

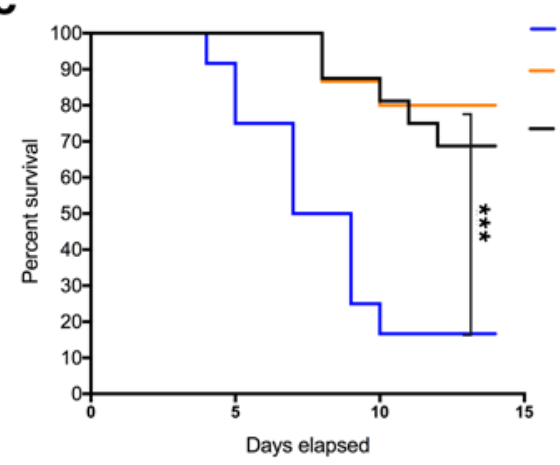

E

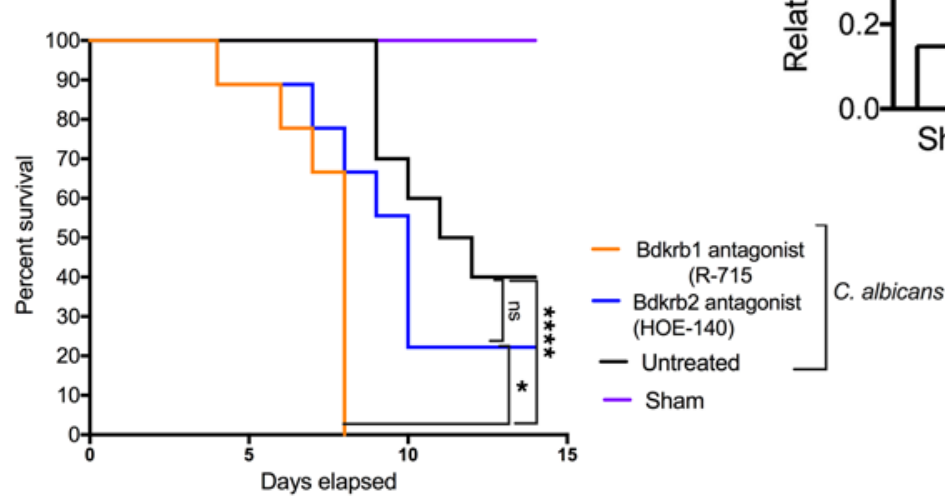

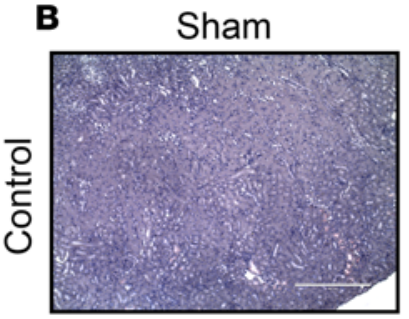
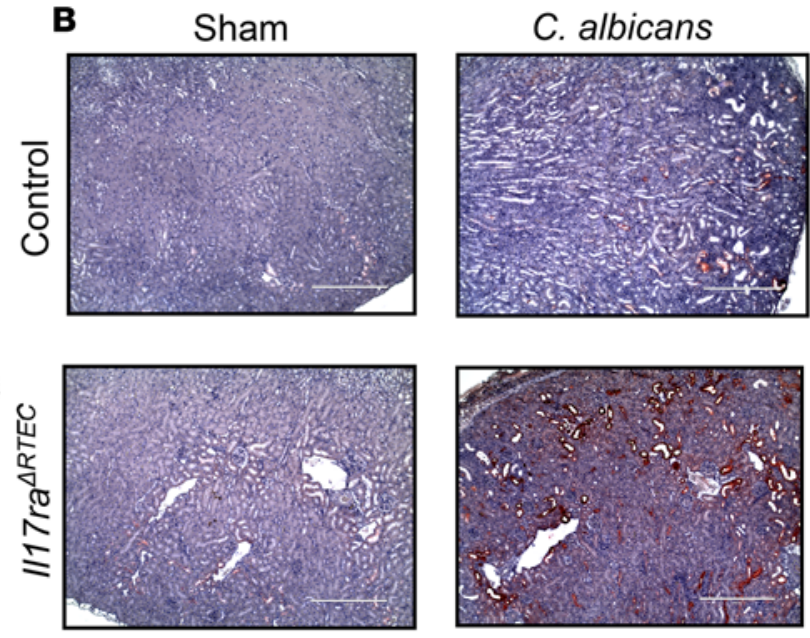

D

Bdkrb1

Bdkrb2
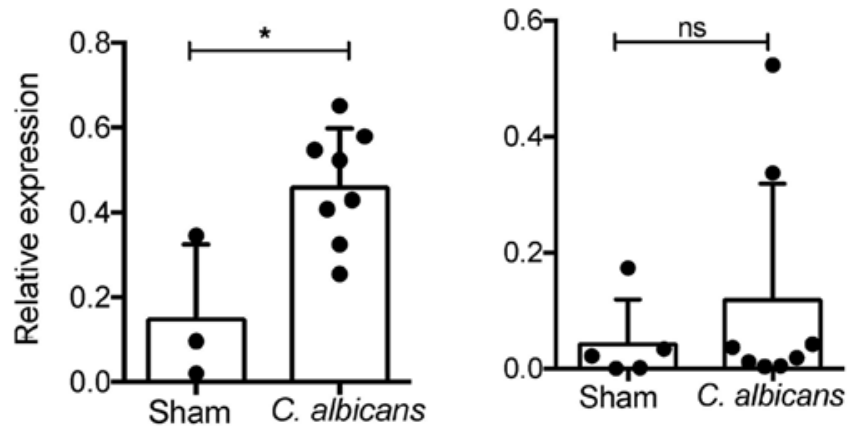

Figure 7. Diminished activation of KKS in the $\boldsymbol{C}$. albicans-infected kidney of $/ 117 \mathbf{r a}^{4 \mathrm{RTEC}}$ mice. Control and $/ 117 \mathrm{ra}{ }^{4 \mathrm{RTEC}}$ mice $(n=8-9)$ were subjected to systemic C. albicans infection. (A) At day 2 p.i., renal transcript expression of $K I k 1$ and $K I k 1 b 26$ was measured by qPCR. Each dot represents an individual mouse, and the data are represented as mean \pm SD. Data were pooled from 2 independent experiments and analyzed by 2-tailed Student's $t$ test. (B) Serial kidney sections $(n=6)$ were stained for KIk1. Original magnification, 100x; scale bars: $400 \mu \mathrm{m}$. Representative photomicrographs from 2 independent experiments. (C) $/ 117 \mathrm{ra}{ }^{4 \mathrm{RTEC}}$ mice $(n=12-15)$ were treated \pm bradykinin ( $\left.300 \mathrm{nmol} / \mathrm{kg} / \mathrm{day}\right)$ starting day -1 (relative to infection) and then daily over 14 days. Control mice were left untreated. Both I/17ra ${ }^{A R T E C}$ and control mice were infected systemically with C. albicans. Sham mice were treated \pm bradykinin ( $n=$ 3-5). Survival was assessed over 14 days. Data are pooled from 4 independent experiments and analyzed by Log-rank (Mantel Cox) test. (D) WT mice ( $n=$ 3-8) were subjected to systemic C. albicans infection. At day 2 p.i., renal transcript expression of Bdkrb1 and Bdkrb2 was quantified by qPCR. Each dot represents individual mouse, and data are represented as mean \pm SD. Data are pooled from 2 independent experiments and analyzed by 2-tailed Student's $t$ test (E) WT mice ( $n=10-11)$ were treated with either Bdkrb1 (R-715; $1 \mathrm{mg} / \mathrm{kg} /$ day) or Bdkrb2 (HOE-140; $1 \mathrm{mg} / \mathrm{kg} /$ day) antagonists or PBS starting day -1 day relative to infection and then daily over 14 days. Sham mice were treated with the antagonists only $(n=3)$. Mice were evaluated for survival over 14 days. The data are pooled from 2 independent experiments and analyzed by Log-rank (Mantel Cox) test. ${ }^{*} P<0.05 ;{ }^{* *} P<0.01 ;{ }^{* *} P<0.001 ;{ }^{* * * *} P<0.0001$.

glomerulus, has no impact on antifungal immunity in the kidney. Nevertheless, we do not rule out the contribution of IL-17R signaling on other cell types in renal protection against disseminated candidiasis. Therefore, additional studies using mice with conditional deletion of Klk1 in RTEC warrants careful consideration.

We show that IL-17RA expression in RTEC plays a minor yet distinguishable delayed role in the renal fungal clearance. This data is in stark contrast to $I 117 \mathrm{ra}^{-/-}$mice, indicating that global IL-17RA signaling, but not RTEC-specific IL-17RA signaling, contributes to control of early fungal growth following systemic C. albicans infection. How IL-17RA signaling in RTEC drives kidney fungal clearance at a delayed time 
point is poorly understood. Our data clearly show that antimicrobial peptides such as $\beta$-defensin 1 and S100a8/9 were significantly downregulated in the kidneys of fungal-infected $I l 17 \mathrm{ra}^{4 \mathrm{RTEC}}$ mice. It is possible that IL-17R signaling in non-RTEC cell types may drive neutrophil influx and early fungal clearance, while late fungal control is mediated by IL-17-driven expression of AMPs from RTEC. Future studies beyond the scope of this manuscript should focus on the sequential events in the kidney that drive early and late fungal clearance in an IL-17-dependent manner.

Our data indicate that Bdkrb1 activation is required for antifungal defense in systemic $C$. albicans infection. This observation is in line with the CNS protective effect of Bdkrb1 in IL-17-dependent experimental autoimmune encephaloymyelitis $(52,53)$. However, these results are quite surprising in context with kidney diseases, since Bdkrb1 activation aggravates renal damage following acute and chronic injury (54-56). It is also not clear why activation of Bdkrb2, which is constitutively expressed in the kidney and is associated with protection against kidney damage, showed a minimal effect on renal defense against systemic infection $(57,58)$. Follow-up studies to dissect signaling events downstream of Bdkrb1 activation in the kidney are required to investigate these unanswered questions. Chiefly, understanding how Bdkrb1 activation alters pro- vs. antiapoptotic signaling events in RTEC following fungal infection may pave the path for developing novel therapeutic approaches to prevent RTEC death and renal insufficiency in the infectious settings.

Overall, our study demonstrates that RTEC-specific IL-17R signaling is critical in regulating KKS in vivo - particularly Klk1 expression and subsequent protection against renal damage in the kidney. Thus, targeting this molecular pathway may provide clinical benefits for patients with disseminated candidiasis in conjunction with antifungal therapy. We also show the essential role of Bdkrb1 in conferring renal protection downstream of the IL-17/Klk1 axis. These observations have potential translational implications, as selective agonists of the Bdkrb1 are in clinical trials. Thus, these data can guide decisions in selecting appropriate pharmacological bradykinin receptor agonists for treating disseminated infection without compromising safety and efficacy.

\section{Methods}

Mice. C57BL/6J (WT) and Cdh16 ${ }^{\text {Cre }}$ mice were purchased from the Jackson Laboratory. $I 117 \mathrm{ra}^{-1-}$ mice were provided by Amgen and bred in-house. $I l 17 \mathrm{ra}^{f l / l}$ mice were provided by Jay Kolls (University of Pittsburgh, Pittsburgh, Pennsylvania, USA) (27). Il17 Cre mice, created by Brigitta Stockinger (The Francis Crick Institute, London, United Kingdom), were crossed to Rosa26eYFP (The Jackson Laboratory) (28). All the experiments included age- and sex-matched controls.

Mouse model of disseminated candidiasis. C. albicans (strains CAF2-1 or HUN96) (provided by Sarah Gaffen, University of Pittsburgh) was grown on yeast extract peptone dextrose (YPD) plates (MilliporeSig $\mathrm{ma})$ at $30^{\circ} \mathrm{C}$ for $18-24$ hours. C. albicans yeast cells were suspended in sterile PBS, and mice were injected via the lateral tail vein with $\mathrm{PBS}$ (sham-infected) or $1 \times 10^{5} \mathrm{CFU} C$. albicans (CAF2-1) or $5 \times 10^{5} \mathrm{CFU} C$. albicans (HUN96). Mice were weighed and monitored daily. Mice were sacrificed if they showed $>20 \%$ weight loss or signs of severe pain or distress.

Evaluation of fungal burden in the kidney. At sacrifice on days 2 and 5 p.i., kidneys were weighed and homogenized in sterile PBS, using a GentleMACS (Miltenyi Biotec). Serial dilutions of organ homogenates were plated on YPD agar with antibiotics (MilliporeSigma), and fungal burden was represented as CFU per gram of tissue.

Measurement of serum BUN. Serum was collected by retro-orbital bleeding at day 7 p.i. Serum BUN levels were measured using Blood Urea Nitrogen Enzymatic kit (Bio Scientific Corp.).

Spleen and kidney cell suspensions. Spleens were harvested from mice and subjected to mechanical dissociation to prepare single cell suspensions, followed by RBC lysis by Mouse Erythrocyte Lysing Kit (R\&D Systems). For kidneys, mice were perfused with PBS containing EDTA (MilliporeSigma) before harvesting the organ. Kidneys were cut into small pieces and digested at $37^{\circ} \mathrm{C}$ in $1 \mathrm{mg} / \mathrm{ml} \mathrm{col-}$ lagenase IV (Worthington) in complete RPMI for 30 minutes. Cells were filtered through 70-mm cell strainers and washed twice in PBS.

Bradykinin and bradykinin receptor antagonists. Mice were injected i.p. with 2001 of bradykinin: 300 $\mathrm{nmol} / \mathrm{kg} /$ day (R\&D Systems) 1 day prior to infection and then daily for 14 days (9). Mice received i.p. injection of Bdkrb1 antagonist (R-715, $1 \mathrm{mg} / \mathrm{kg} /$ day, R\&D Systems), Bdkrb2 antagonist (HOE-140, $1 \mathrm{mg} /$ $\mathrm{kg} /$ day, R\&D Systems) starting day -1 (relative to infection) and daily for 14 days, as described previously (9). Control mice received equal volume of PBS. 
$H \& E$ and immunohistochemical staining. For kidney histology, formalin-fixed paraffin embedded kidney sections were subjected to $H \& E$ staining.

Immunohistochemal staining was done on formalin-fixed paraffin embedded sections. Sections were rehydrated, and antigen retrieval was performed with heated citrate. Primary antibodies against the following proteins were used: Klk1 (LS-C312857-100, LifeSpan Biosciences), NGAL (AF1757, R\&D Biosystems), and KIM1 (Ab47635, Abcam). Secondary antibodies used were horseradish peroxidase-coupled antibodies (705-035-003 and 111-035-144, Jackson ImmunoResearch). Images were obtained with EVOS FL Auto microscope (Invitrogen).

Flow cytometry. At sacrifice on days 2, 5, or 7 p.i., as indicated in the text, kidneys were harvested following perfusion with PBS. The single cell suspensions were slowly layered over 5-ml Lympholyte (Cedarlane). The tubes were spun for 1,300 $\mathrm{g}$ for 30 minutes at room temperature. The cell layer at the interface of the media and Lympholyte was collected and washed twice with PBS and used for FACS staining. To identify the proliferating IL-17-producing cells, cells were stained with the following fluorescent conjugated antibodies: CD45 (30-F11, BioLegend), TCR $\beta$ (H57-597, BioLegend), and TCR $\delta$ (GL3, BioLegend) and CD4 (GK1.5, eBiosciences), followed by intracellular staining with Ki-67 (B56, BD Biosciences). For flow cytometry staining of kidney-infiltrating inflammatory cells and tubular epithelial cells, cell suspensions were stained with antibodies against CD45 (30-F11), Ly6G (IA8; 1A8-Ly6g), CD11b (M1/70), F4/80 (BM8), CD3 (145-2C11), and NK1.1 (PK136; all from eBiosciences). For the detection of IFN $\gamma$ production by NK cells in spleen suspensions, cells were first stained with antibodies against CD45, CD3 (145-2C11), CD69 (H1.2F3), NKp46 (29A1.4), and CD49b (DX5; all from BioLegend) and were subsequently stained with anti-IFN $\gamma$ and antiGM-CSF antibodies (BD Pharmingen) after fixation and permeabilization using intracellular cytokine staining kit (BD Pharmingen, San Jose CA). For the detection of IL-17RA expression by NK cells in BM cell suspensions, cells were stained with antibodies against CD45, CD3, CD122 (TM- $\beta 1)$, CD94 (18d3), and CD49b (all from BioLegend) and IL-17R (PAJ-17R, Thermo Fisher Scientific). Data were acquired on a BD LSRFortessa cytometer (BD Biosciences) and analyzed with FlowJo (Tree Star Inc.).

To determine the frequency of dead and active Caspase $3^{+}$cells, perfused kidneys were harvested at day 7 p.i. Single cell suspension were prepared by treating minced kidney tissues in $1 \mathrm{mg} / \mathrm{ml}$ collagenase IV (Worthington) in complete RPMI for 30 minutes. Total kidney cells were surface stained with anti-CD45 and anti-CD133 antibodies (13A4, eBiosciences). To detect apoptotic cells and active Caspase-3 expression, cells were stained with 7-AAD (BD Pharmingen) and CaspGLOW Fluroscein active Caspase-3 staining kit (Thermo Fisher Scientific), respectively, as per the manufacturer's instructions.

For determination of absolute numbers of IL-17-producing cells, $50 \mu 1$ of CountBright absolute counting beads (Thermo Fisher Scientific) were added to $400 \mu 1$ stained cell samples. The samples were analyzed on BD LSRFortessa cytometer (BD Biosciences). The absolute number IL-17-producing cells in samples were calculated by comparing the ratio of bead events to cell events.

RNA extraction and real-time PCR. For real-time PCR analyses, RNA from homogenized kidneys of sham or infected mice was extracted using the RNAEasy Mini kit (Qiagen). RNA was reverse-transcribed using the Superscript III First-strand kit (Invitrogen). Real-time PCR was performed using PerfeCTa SYBR Green FastMix ROX (Quanta BioSciences) on a 7300 Real-Time PCR System (Applied Biosystems). Primers were purchased from Quantitect (Qiagen). The expression of each gene was normalized to Gapdh.

Statistics. Data are presented as mean $\pm \mathrm{SD}$, as indicated in the legend of each figure. The significance of the differences between groups was evaluated using unpaired 2-tailed $t$ test or 1-way ANOVA wherever appropriate; $P<0.05$ was considered significant. Survival curve data are presented as Kaplan-Maier plots with a log rank test to compare susceptibility between experimental groups. In figures, asterisks are used to denote statistical significance $\left({ }^{*} P<0.05 ;{ }^{*} P<0.01 ;{ }^{* * *} P<0.001 ;{ }^{* * * *} P<0.0001\right)$. Statistical analysis was performed in GraphPad PRISM 7. All experiments were performed at least twice to ensure reproducibility.

Study approval. The animal protocols used in this work were evaluated and approved by the University of Pittsburgh IACUC (protocols 14094427 and 14043544) and adhered to the guidelines in the Guide for the Care and Use of Laboratory Animals of the NIH (National Academies Press, 2011).

\section{Author contributions}

$\mathrm{KR}, \mathrm{CVJ}, \mathrm{AHV}, \mathrm{BMC}$, and PSB conducted experiments and acquired and analyzed data; JKK provided reagents; KR and PSB designed research studies and wrote the manuscript with input from all of the coauthors. 


\section{Acknowledgments}

This work is supported by NIH grant DK104680 to PSB. The authors thank the Unified Flow Core, Department of Immunology, University of Pittsburgh for flow cytometry, as well as Department of Medicine, University of Pittsburgh Start-up fund (to PSB). We thank Sarah Gaffen and Mandy McGeachy for helpful suggestions and discussions.

Address correspondence to: Partha Sarathi Biswas, S725 BST, Division of Rheumatology and Clinical Immunology, University of Pittsburgh, Pittsburgh, Pennsylvania 15261, USA. Phone: 412.648.8708; Email:psb13@pitt.edu.

1. Brown GD, Netea MG. Exciting developments in the immunology of fungal infections. Cell Host Microbe. 2012;11(5):422-424

2. Pfaller MA, Diekema DJ. Epidemiology of invasive candidiasis: a persistent public health problem. Clin Microbiol Rev. 2007;20(1):133-163.

3. Brown GD, Denning DW, Gow NA, Levitz SM, Netea MG, White TC. Hidden killers: human fungal infections. Sci Transl Med. 2012;4(165):165rv13.

4. González de Molina FJ, León C, Ruiz-Santana S, Saavedra P, CAVA I Study Group. Assessment of candidemia-attributable mortality in critically ill patients using propensity score matching analysis. Crit Care. 2012;16(3):R105.

5. Lionakis MS. New insights into innate immune control of systemic candidiasis. Med Mycol. 2014;52(6):555-564.

6. Taur Y, Cohen N, Dubnow S, Paskovaty A, Seo SK. Effect of antifungal therapy timing on mortality in cancer patients with candidemia. Antimicrob Agents Chemother. 2010;54(1):184-190.

7. Huang W, Na L, Fidel PL, Schwarzenberger P. Requirement of interleukin-17A for systemic anti-Candida albicans host defense in mice. J Infect Dis. 2004;190(3):624-631.

8. Bär E, Whitney PG, Moor K, Reis e Sousa C, LeibundGut-Landmann S. IL-17 regulates systemic fungal immunity by controlling the functional competence of NK cells. Immunity. 2014;40(1):117-127.

9. Ramani K, et al. The Kallikrein-Kinin System: A Novel Mediator of IL-17-Driven Anti-Candida Immunity in the Kidney. PLoS Pathog. 2016;12(11):e1005952.

10. van de Veerdonk FL, et al. Differential effects of IL-17 pathway in disseminated candidiasis and zymosan-induced multiple organ failure. Shock. 2010;34(4):407-411.

11. Ho AW, Gaffen SL. IL-17RC: a partner in IL-17 signaling and beyond. Semin Immunopathol. 2010;32(1):33-42.

12. Gaffen SL. Structure and signalling in the IL-17 receptor family. Nat Rev Immunol. 2009;9(8):556-567.

13. Conti HR, et al. IL-17 Receptor Signaling in Oral Epithelial Cells Is Critical for Protection against Oropharyngeal Candidiasis. Cell Host Microbe. 2016;20(5):606-617.

14. Ramani K, Pawaria S, Maers K, Huppler AR, Gaffen SL, Biswas PS. An essential role of interleukin-17 receptor signaling in the development of autoimmune glomerulonephritis. J Leukoc Biol. 2014;96(3):463-472.

15. Xu W, Solis NV, Ehrlich RL, Woolford CA, Filler SG, Mitchell AP. Activation and alliance of regulatory pathways in C. albicans during mammalian infection. PLoS Biol. 2015;13(2):e1002076.

16. Conti HR, et al. Th17 cells and IL-17 receptor signaling are essential for mucosal host defense against oral candidiasis. $J$ Exp Med. 2009;206(2):299-311.

17. Hillmeister P, Persson PB. The Kallikrein-Kinin system. Acta Physiol (Oxf). 2012;206(4):215-219.

18. Kakoki M, Smithies O. The kallikrein-kinin system in health and in diseases of the kidney. Kidney Int. 2009;75(10):1019-1030.

19. Jozwiak L, Drop A, Buraczynska K, Ksiazek P, Mierzicki P, Buraczynska M. Association of the human bradykinin B2 receptor gene with chronic renal failure. Mol Diagn. 2004;8(3):157-161.

20. Baboolal K, et al. Association of the angiotensin I converting enzyme gene deletion polymorphism with early onset of ESRF in PKD1 adult polycystic kidney disease. Kidney Int. 1997;52(3):607-613.

21. Harden PN, et al. Polymorphisms in angiotensin-converting-enzyme gene and progression of IgA nephropathy. Lancet. 1995;345(8964):1540-1542.

22. Liu K, et al. Kallikrein genes are associated with lupus and glomerular basement membrane-specific antibody-induced nephritis in mice and humans. J Clin Invest. 2009;119(4):911-923.

23. Louis K, Hertig A. How tubular epithelial cells dictate the rate of renal fibrogenesis? World J Nephrol. 2015;4(3):367-373

24. Hato T, El-Achkar TM, Dagher PC. Sisters in arms: myeloid and tubular epithelial cells shape renal innate immunity. Am J Physiol Renal Physiol. 2013;304(10):F1243-F1251.

25. Lionakis MS, Lim JK, Lee CC, Murphy PM. Organ-specific innate immune responses in a mouse model of invasive candidiasis. J Innate Immun. 2011;3(2):180-199.

26. Conti HR, et al. Oral-resident natural Th17 cells and $\gamma \delta \mathrm{T}$ cells control opportunistic Candida albicans infections. $J$ Exp Med. 2014;211(10):2075-2084.

27. Kashem SW, Riedl MS, Yao C, Honda CN, Vulchanova L, Kaplan DH. Nociceptive Sensory Fibers Drive Interleukin-23 Production from CD301b+ Dermal Dendritic Cells and Drive Protective Cutaneous Immunity. Immunity. 2015;43(3):515-526.

28. Hirota K, et al. Fate mapping of IL-17-producing T cells in inflammatory responses. Nat Immunol. 2011;12(3):255-263.

29. Dejima T, et al. Protective role of naturally occurring interleukin-17A-producing $\gamma \delta \mathrm{T}$ cells in the lung at the early stage of systemic candidiasis in mice. Infect Immun. 2011;79(11):4503-4510.

30. Timoshanko JR, Holdsworth SR, Kitching AR, Tipping PG. IFN-gamma production by intrinsic renal cells and bone marrow-derived cells is required for full expression of crescentic glomerulonephritis in mice. J Immunol. 2002;168(8):4135-4141.

31. Timoshanko JR, Kitching AR, Semple TJ, Holdsworth SR, Tipping PG. Granulocyte macrophage colony-stimulating factor expression by both renal parenchymal and immune cells mediates murine crescentic glomerulonephritis. J Am Soc Nephrol. 
2005;16(9):2646-2656

32. Ruth AJ, Kitching AR, Semple TJ, Tipping PG, Holdsworth SR. Intrinsic renal cell expression of CD40 directs Th1 effectors inducing experimental crescentic glomerulonephritis. J Am Soc Nephrol. 2003;14(11):2813-2822.

33. Zhao X, et al. JNK1 negatively controls antifungal innate immunity by suppressing CD23 expression. Nat Med. 2017;23(3):337-346

34. Tan RJ, Zhou D, Liu Y. Signaling Crosstalk between Tubular Epithelial Cells and Interstitial Fibroblasts after Kidney Injury. Kidney Dis (Basel). 2016;2(3):136-144.

35. Shao X, Somlo S, Igarashi P. Epithelial-specific Cre/lox recombination in the developing kidney and genitourinary tract. $J A m$ Soc Nephrol. 2002;13(7):1837-1846.

36. Kumar P, et al. Intestinal Interleukin-17 Receptor Signaling Mediates Reciprocal Control of the Gut Microbiota and Autoimmune Inflammation. Immunity. 2016;44(3):659-671.

37. MacCallum DM, Castillo L, Brown AJ, Gow NA, Odds FC. Early-expressed chemokines predict kidney immunopathology in experimental disseminated Candida albicans infections. PLoS ONE. 2009;4(7):e6420.

38. Moeller MJ, Sanden SK, Soofi A, Wiggins RC, Holzman LB. Podocyte-specific expression of cre recombinase in transgenic mice. Genesis. 2003;35(1):39-42.

39. Kai T, et al. Kidney-specific knockout of Sav1 in the mouse promotes hyperproliferation of renal tubular epithelium through suppression of the Hippo pathway. J Pathol. 2016;239(1):97-108.

40. Chen K, et al. IL-17 Receptor Signaling in the Lung Epithelium Is Required for Mucosal Chemokine Gradients and Pulmonary Host Defense against K. pneumoniae. Cell Host Microbe. 2016;20(5):596-605.

41. Whibley N, et al. Delinking CARD9 and IL-17: CARD9 Protects against Candida tropicalis Infection through a TNF- $\alpha$-Dependent, IL-17-Independent Mechanism. J Immunol. 2015;195(8):3781-3792.

42. Conti HR, Whibley N, Coleman BM, Garg AV, Jaycox JR, Gaffen SL. Signaling through IL-17C/IL-17RE is dispensable for immunity to systemic, oral and cutaneous candidiasis. PLoS One. 2015;10(4):e0122807.

43. Domínguez-Andrés J, Feo-Lucas L, Minguito de la Escalera M, González L, López-Bravo M, Ardavín C. Inflammatory Ly6ChighMonocytes Protect against Candidiasis through IL-15-Driven NK Cell/Neutrophil Activation. Immunity. 2017;46(6):1059-1072.e4.

44. Quintin J, et al. Differential role of NK cells against Candida albicans infection in immunocompetent or immunocompromised mice. Eur J Immunol. 2014;44(8):2405-2414.

45. Huppler AR, Conti HR, Hernández-Santos N, Darville T, Biswas PS, Gaffen SL. Correction: Role of Neutrophils in IL-17-Dependent Immunity to Mucosal Candidiasis. J Immunol. 2015;194(3):1382.

46. Arancia G, et al. Noninhibitory binding of human interleukin-2-activated natural killer cells to the germ tube forms of Candida albicans. Infect Immun. 1995;63(1):280-288.

47. Arancia G, et al. Interaction between human interleukin-2-activated natural killer cells and heat-killed germ tube forms of Candida albicans. Cell Immunol. 1998;186(1):28-38

48. Gaforio JJ, Ortega E, Algarra I, Serrano MJ, Alvarez de Cienfuegos G. NK cells mediate increase of phagocytic activity but not of proinflammatory cytokine (interleukin-6 [IL-6], tumor necrosis factor alpha, and IL-12) production elicited in splenic macrophages by tilorone treatment of mice during acute systemic candidiasis. Clin Diagn Lab Immunol. 2002;9(6):1282-1294.

49. Voigt J, et al. Human natural killer cells acting as phagocytes against Candida albicans and mounting an inflammatory response that modulates neutrophil antifungal activity. J Infect Dis. 2014;209(4):616-626.

50. Murciano C, Villamón E, O’Connor JE, Gozalbo D, Gil ML. Killed Candida albicans yeasts and hyphae inhibit gamma interferon release by murine natural killer cells. Infect Immun. 2006;74(2):1403-1406.

51. Rieber N, et al. Pathogenic fungi regulate immunity by inducing neutrophilic myeloid-derived suppressor cells. Cell Host Microbe. 2015;17(4):507-514.

52. Schulze-Topphoff U, et al. Activation of kinin receptor B1 limits encephalitogenic T lymphocyte recruitment to the central nervous system. Nat Med. 2009;15(7):788-793.

53. Göbel K, et al. Blockade of the kinin receptor B1 protects from autoimmune CNS disease by reducing leukocyte trafficking. $J$ Autoimmun. 2011;36(2):106-114.

54. Wang PH, et al. Deletion of bradykinin B1 receptor reduces renal fibrosis. Int Immunopharmacol. 2009;9(6):653-657.

55. Klein J, et al. Delayed blockade of the kinin B1 receptor reduces renal inflammation and fibrosis in obstructive nephropathy. FASEB J. 2009;23(1):134-142.

56. Pereira RL, et al. Bradykinin receptor 1 activation exacerbates experimental focal and segmental glomerulosclerosis. Kidney Int. 2011;79(11):1217-1227.

57. Schanstra JP, et al. In vivo bradykinin B2 receptor activation reduces renal fibrosis. J Clin Invest. 2002;110(3):371-379.

58. Maltais I, Bachvarova M, Maheux P, Perron P, Marceau F, Bachvarov D. Bradykinin B2 receptor gene polymorphism is associated with altered urinary albumin/creatinine values in diabetic patients. Can J Physiol Pharmacol. 2002;80(4):323-327. 